\title{
Study of Order Parameters Coupling in Uniaxial Ferroelectrics on the Basis of Pyroelectric and Dilatometric Measurements
}

\author{
M. GAŁAzZKA ${ }^{a, *}$ AND P. ZIELIŃSKI ${ }^{a, b}$ \\ ${ }^{a}$ The H. Niewodniczański Institute of Nuclear Physics, Polish Academy of Sciences \\ E. Radzikowskiego 152, 31-342 Cracow, Poland \\ ${ }^{b}$ Institute of Physics, Cracow University of Technology, Podchorążych 1, 30-084 Cracow, Poland \\ A method is devised of extracting the explicit form of the coupling between the primary and secondary order \\ parameters with the use of experimental data and respecting classical and non-classical values of the effective \\ critical exponents. The corresponding equation of state stems from the Ising model on a compressible lattice \\ treated within the mean field approximation supplemented with terms ascertaining scaling invariance in the \\ vicinity of the critical point. The theory is exemplified by the molecular ferroelectric crystals $\left(\mathrm{CH}_{3} \mathrm{NH}_{3}\right)_{5} \mathrm{Bi}_{2} \mathrm{Cl}_{11}$ \\ (MAPCB) and $\left(\mathrm{CH}_{3} \mathrm{NH}_{3}\right)_{5} \mathrm{Bi}_{2} \mathrm{Br}_{11}$ (MAPBB).
}

PACS numbers: $64.10 .+\mathrm{h}, 64.60 . \mathrm{Bd}, 62.20 . \mathrm{dq}$

\section{Introduction}

The full description of the temperature behaviour of spontaneous values of order parameters in the phase transitions involve the critical phenomena close to the transition point, whenever the transition is continuous, and there is a saturation at lower temperatures. The latter region is particularly interesting for applications. The simplest models allow one to construct the required equations of state but the coupling with a number of secondary order parameters modifies significantly their predictions. In the present paper we consider a two-state pseudospin model, i.e. the Ising model on a deformable lattice, and show that the knowledge of the temperature dependence of the primary order parameter determines the behaviour of the secondary order parameter up to a single constant and, moreover, allows one to find the analytical form of the coupling function.

The Ising model [1-5] describes behaviour of materials undergoing the second order phase transitions. The thermodynamics of this model is well known in the vicinity of the phase transition giving non-classical critical exponents depending on the dimension of the lattice (for example see Refs. [4, 5]). When treated within the mean field approximation, it can be solved at all temperatures but then gives classical critical exponents. The present authors will show how to modify the mean field treatment to obtain a critical behaviour compatible with the scaling hypothesis [5-8] and at the same time the saturation at lower temperatures. The scaling equations of

* corresponding author; e-mail: Miroslaw.Galazka@ifj.edu.pl state provide a correct description of real materials undergoing the second order phase transitions even if the full equilibrium is not attained because of a finite rate of temperature changes (for example see [8-12]). Then all of materials parameters, as, for example, the ratio of the inverse Curie-Weiss constants, $\Gamma_{-} / \Gamma_{+}$, the effective critical exponents, are a result of interplay between static and dynamical phenomena. Therefore, the corresponding critical exponents are usually different from those implied by universality classes because their values result from the materials properties (as a heat capacity, a relaxation time, etc.) and from the experimental conditions (as a finite temperature rate, a measuring frequency, a finite size of sample, etc.) [10, 13-15].

The main drawback of the Ising model is that the order parameter saturation in temperatures is much slower than it takes place in real materials $[12,16,17]$. Therefore, this is an indication that one should take into consideration one more degree of freedom, i.e. a coupling with a secondary order parameter.

Here we consider an equation of state which involves the coupling of the electric polarization $\langle\sigma\rangle$, being the primary order parameter in ferroelectric phase transitions, with a secondary order parameter, in our case a mechanical strain $\varepsilon$ as presented in Sect. 2. Section 3 contains essential relations resulting from the equation of state. These results are exemplified by the experimental data of ferroelectrics MAPCB and MAPBB in Sect. 4 .

\section{Compressible pseudospin model}

The mean field Hamiltonian of a two state Ising pseudospin model on a compressible lattice reads [16]: 


$$
\begin{gathered}
H_{\mathrm{MFA}}=-\sum_{i}[J(\varepsilon)\langle\sigma\rangle+h] \sigma_{i}+\frac{1}{2} \sum_{i} J(\varepsilon)\langle\sigma\rangle^{2} \\
+\frac{1}{2} c_{\mathrm{el}} \varepsilon^{2}-s \varepsilon
\end{gathered}
$$

$\sigma_{i}$ is the Ising variable at the site number $i,\langle\sigma\rangle$ - its average over all the sites, i.e. the spontaneous polarization, $\varepsilon$ is the strain describing the deformation of the material entailed by the polarization $\langle\sigma\rangle$. The function $J(\varepsilon)$ is a coupling function and $c_{\mathrm{el}}$ - the elastic constant. The external field $\mathrm{h}$ is conjugated to the primary order parameter $\langle\sigma\rangle$ and $\mathrm{s}$ is the stress (the field conjugated to the secondary order parameter $\varepsilon$ ). The external fields $\mathrm{h}$ and $\mathrm{s}$, and the elastic constant $c_{\mathrm{el}}$ are given in the units of energy.

Using the Hamiltonian of Eq. (1) one obtains the free energy density

$$
F \equiv-k T \ln \left(\sum_{\sigma_{i}= \pm 1} \exp \left(-\frac{H_{\mathrm{MFA}}}{k T}\right)\right)
$$

which can be written in the explicit form

$$
\begin{aligned}
& F(\langle\sigma\rangle, \varepsilon ; T, h, s)=\frac{1}{2} c_{\mathrm{el}} \varepsilon^{2}+\frac{1}{2} J(\varepsilon)\langle\sigma\rangle^{2} \\
& \quad-k T \ln \left(2 \cosh \left(\frac{J(\varepsilon)\langle\sigma\rangle+h}{k T}\right)\right)-s \varepsilon .
\end{aligned}
$$

The equilibrium state of the system under external fields is defined by the necessary conditions of minimum of the free energy density: $\partial F / \partial\langle\sigma\rangle=0$ and $\partial F / \partial \varepsilon=0$

$$
h=-J(\varepsilon)\langle\sigma\rangle+k T \operatorname{artanh}\langle\sigma\rangle
$$

and

$$
s=c_{\mathrm{el}} \varepsilon-\frac{1}{2}\langle\sigma\rangle^{2} \frac{\partial J(\varepsilon)}{\partial \varepsilon},
$$

where $\operatorname{artanh}(x)$ is the area hyperbolic tangent function of a variable $x$.

Equations (4a) and (4b) are the equations of state in the compressible pseudospin model in the mean field approximation. The explicit form of the coupling function $J(\varepsilon)$ is usually not known. As long as the strain is not large, $\varepsilon \ll 1$, the function $J(\varepsilon)$ can be approximated by a power series

$$
J(\varepsilon)=J_{0}+J_{1} \varepsilon+J_{2} \varepsilon^{2}+\ldots,
$$

where $J_{0}=k T_{\mathrm{C}}$ is the Ising exchange parameter on a rigid lattice.

The power expansions of Eqs. (4a) and (4b) in the variables $\langle\sigma\rangle$ and $\varepsilon$ reproduce the Landau equations of state valid in the vicinity of the critical point, whereas the full form of these equations describes the behaviour of the system down to the lowest temperatures.

\section{The coupling function and the secondary order parameter}

Knowing the temperature dependences of the spontaneous polarization $\langle\sigma\rangle$ and of the mechanical stress $\varepsilon$ from experimental measurements one can obtain the coupling function $J(\varepsilon)$ resulting from Eq. (4a) at the zero field, $h=0$ :

$$
J(\varepsilon)=k T \frac{\operatorname{artanh}\langle\sigma\rangle}{\langle\sigma\rangle}
$$

and the derivative of the coupling function with respect to the strain, $\partial J(\varepsilon) / \partial \varepsilon$, following from Eq. (4b) at zero stress, $s=0$

$$
\frac{\partial J(\varepsilon)}{\partial \varepsilon}=\frac{2 c_{\mathrm{el}} \varepsilon}{\langle\sigma\rangle^{2}} .
$$

If the elastic constant $c_{\mathrm{el}}$ is not known, the function $\partial J / \partial \varepsilon$ is calculated from Eq. (7) up to a multiplicative factor.

One more relation between the spontaneous polarization $\langle\sigma\rangle$ and the spontaneous strain $\varepsilon$ can be derived from the equations of state given by Eqs. (4a) and (4b), where the coupling function is entirely eliminated. The relation reads

$$
\begin{gathered}
\frac{\varepsilon^{2} c_{\mathrm{el}}}{k}=-\left.T \ln \left((1+\langle\sigma\rangle)^{1+\langle\sigma\rangle / 2}(1-\langle\sigma\rangle)^{1-\langle\sigma\rangle / 2}\right)\right|_{\mathrm{T}_{\mathrm{C}}} ^{\mathrm{T}} \\
+\int_{\mathrm{T}_{\mathrm{C}}}^{\mathrm{T}} \ln \left((1+\langle\sigma\rangle)^{1+\langle\sigma\rangle}(1-\langle\sigma\rangle)^{1-\langle\sigma\rangle}\right) \mathrm{d} T^{\prime},
\end{gathered}
$$

where $k$ is the Boltzmann constant. Equation (8) may serve as a test of the correctness of the assumptions adopted. One can extract the temperature dependence of the strain $\varepsilon$ directly from the spontaneous polarization $\langle\sigma\rangle$ with the use of Eq. (8). The dependence $\partial \varepsilon^{2} / \partial \mathrm{T}$ on the spontaneous polarization $\langle\sigma\rangle$ has been given in Ref. [16]. However, Eq. (8) requires fewer mathematical operations than analogous formula given in Ref. [16] to obtain the strain $\varepsilon$ from the spontaneous polarization data $\langle\sigma\rangle$.

It is well known that the effective critical exponents may differ from the classical ones inferred by the above mean field treatment (for example see Refs. [8, 10, 12-14, 17]). In Ref. [17] formulae analogous to those $((2)-(7))$ have been given. In what follows we analyze the experimental data for two ferroelectric compounds within the present mean field theory.

\section{Application to experimental data}

The theoretical considerations of previous section will be exemplified on the uniaxial methylammonium ferroelectric salts $\left(\mathrm{CH}_{3} \mathrm{NH}_{3}\right)_{5} \mathrm{Bi}_{2} \mathrm{Cl}_{11}$ and $\left(\mathrm{CH}_{3} \mathrm{NH}_{3}\right)_{5} \mathrm{Bi}_{2} \mathrm{Br}_{11}$ abbreviated MAPCB and MAPBB, respectively. Molecular ferroelectrics MAPCB and MAPBB undergo the second order phase transition at $T_{\mathrm{C}}=307.65 \mathrm{~K}[8,10,18]$ and at $T_{\mathrm{C}}=311.52 \mathrm{~K}[8,10]$, respectively. The symmetry relation is $P c a b \rightarrow P c a 2_{1}$ in both cases $[8,10,16]$. The mechanism of the phase transitions involves ordering of one of three types of the methylammonium cations $[8,10,12,13,16,19-21]$.

Numerous studies of the spontaneous polarization $\langle\sigma\rangle$ confirm the power law behaviour with the non-classical and at the same time non-universal exponents $\beta_{\text {eff }}[8,10$, $12,22,23]$ which are $0.379 \pm 0.020$ for MAPCB and $0.375 \pm 0.020$ for MAPBB. The effective exponents $\gamma_{\text {eff }}$ are close to the classical one and they are equal 
to $0.985 \pm 0.030$ for MAPCB $[8-10,14,18,24]$ and $0.989 \pm 0.030$ for MAPBB [8-10, 14, 18, 24].

The pyroelectric current was measured by a KEITHLEY 617 electrometer with temperature rate $0.5 \mathrm{~K} / \mathrm{min}$. The dilatometric measurement were performed by a thermomechanical analyser Perkin Elmer TMA-7 with temperature scanning rate $4 \mathrm{~K} / \mathrm{min}$. The overall errors in measurements were about $5 \%$. The dimensions of the samples were of order $5 \times 3 \times 1 \mathrm{~mm}^{3}$. The preparation of the crystals and the experimental details are given in $[10,25]$.

Figure 1 presents the experimental spontaneous strain $\varepsilon$ for MAPCB (solid curve) and MAPBB (dotted curve). The spontaneous "pancake" strain $\varepsilon$, defined in Ref. [26] as $\varepsilon \equiv-\left(2 \varepsilon_{33}-\varepsilon_{11}-\varepsilon_{22}\right) / \sqrt{3}$ turns out to be the strongest spontaneous strain appearing in the low temperature phase [16], has been extracted from the dilatometric measurements [27]. The decrease and the sudden increase of the strain $\varepsilon$ below $\tau_{\text {sat }}=-0.35$ for ferroelectric MAPCB is connected with an anomaly and an ordering of another methylammonium cations at $170 \mathrm{~K}$ $[16,19,20,26,28,29]$. The similar anomaly occurs at $77 \mathrm{~K}$ for ferroelectric MAPBB [20, 26, 30].

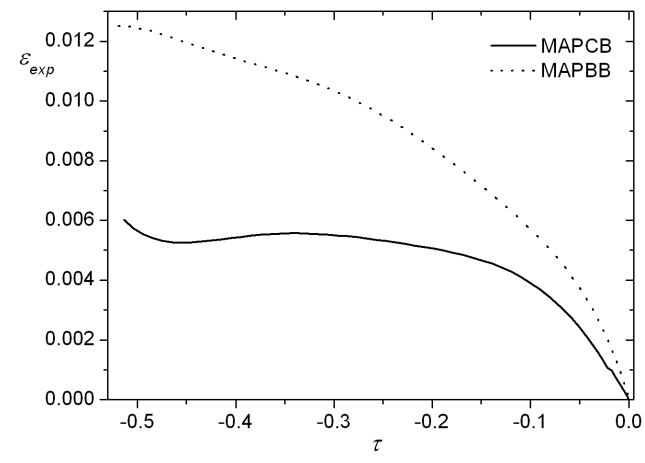

Fig. 1. Experimental dependences of the strain $\varepsilon$ on the reduced temperature $\tau$ below the phase transition points at zero biasing field and stress-free samples for ferroelectrics MAPCB (solid curve) and MAPBB (dotted curve).

Figures $2 \mathrm{a}$ and $2 \mathrm{~b}$ show some experimental data (open rhombus) of the spontaneous polarization $\langle\sigma\rangle$ for ferroelectrics MAPCB and MAPBB, respectively. The solid curves are results of the solution of Eqs. (4a) and (4b) at zero biasing fields, $h=0$ and $s=0$, with the coefficients presented in Table. As one can see, the agreements between experimental and theoretical curves are very good for both molecular crystals as well close to the phase transition points $T_{\mathrm{C}}$ as far below these points. The coefficients $J_{0}, J_{1}$ and $J_{2}$, given in Table, have been obtained on the basis of Eqs. (5), (6) and on the spontaneous polarization data for both molecular crystals.

The theoretical quantities $\varepsilon c_{\mathrm{el}}^{1 / 2}$ divided by square root of Boltzmann's constant $k$ obtained from Eq. (8) are shown in Fig. 3 for MAPCB (solid line) and MAPBB (dotted line). Comparing dependences of the strains $\varepsilon$

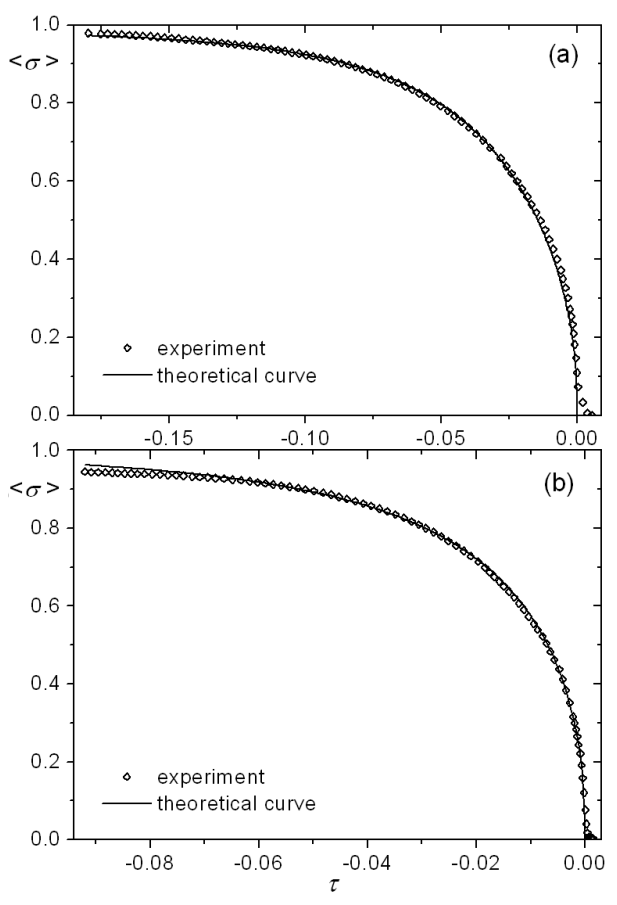

Fig. 2. Some experimental points and fitted curves to the data of the spontaneous polarization $\langle\sigma\rangle$ for (a) MAPCB and (b) MAPBB crystals. Fitted parameters are given in Table.

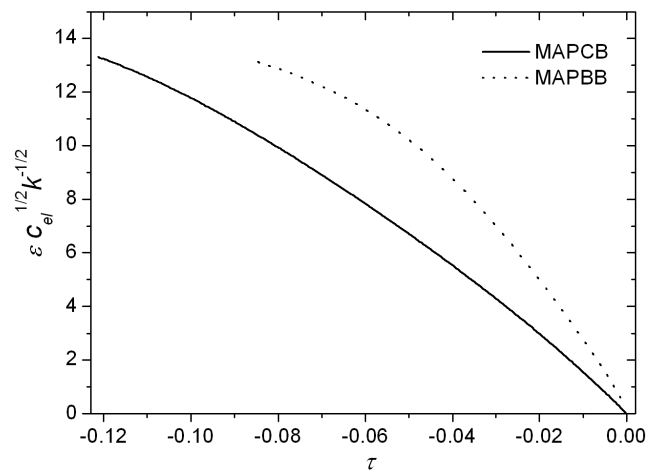

Fig. 3. Theoretical quantity $\varepsilon c_{\mathrm{el}}^{1 / 2}$ multiplied by factor $k^{-1 / 2}$ obtained from measurements of the spontaneous polarization $\langle\sigma\rangle$ for ferroelectrics MAPCB (solid curve) and MAPBB (dotted curve).

on the temperature, shown in Fig. 1, with the temperature dependences of $\varepsilon c_{\mathrm{el}}^{1 / 2}$, shown in Fig. 3, one gets the values of the elastic constants $c_{\mathrm{el}}^{(1)}$, presented in Table, for both crystals. The obtained elastic constants $c_{\mathrm{el}}^{(1)}$ are weak-temperature dependent therefore we gave the mean values of them. Moreover, the elastic constants $c_{\mathrm{el}}^{(2)}$, obtained by comparing the dependences $J(\varepsilon)$ and $\partial J / \partial \varepsilon$, are also shown in Table for MAPCB and MAPBB. The resulting elastic constants are of order $10^{10} \div 10^{11} \mathrm{~N} \mathrm{~m}^{-2}$ and they are plausible for a macroscopic strains [16]. 


\section{TABLE}

Parameters of coupling function $J(\varepsilon)$ and elastic constants obtained in two different ways: $c_{\mathrm{el}}^{(1)}$ - from comparison of experimental spontaneous strain $\varepsilon_{\exp }(\tau)$ with theoretical quantity $\varepsilon_{\text {theo }}(\tau) c_{\mathrm{el}}^{1 / 2}$ obtained from spontaneous polarization data, and $c_{\mathrm{el}}^{(2)}$ - from comparison of dependences $J(\varepsilon)$ and $\partial J / \partial \varepsilon$. All of presented parameters are given in units of temperature.

\begin{tabular}{c|c|c}
\hline \hline & MAPCB & MAPBB \\
\hline$J_{0} / k[\mathrm{~K}]$ & $307.65 \pm 0.03$ & $311.52 \pm 0.03$ \\
$J_{1} / k[\mathrm{~K}]$ & $(1.30 \pm 0.07) \times 10^{5}$ & $(2.49 \pm 0.13) \times 10^{5}$ \\
$J_{2} / k[\mathrm{~K}]$ & $(5.68 \pm 0.28) \times 10^{6}$ & $(1.43 \pm 0.07) \times 10^{6}$ \\
$c_{\mathrm{el}}^{(1)} / k[\mathrm{~K}]$ & $(1.47 \pm 0.07) \times 10^{7}$ & $(1.97 \pm 0.10) \times 10^{7}$ \\
$c_{\mathrm{el}}^{(2)} / k[\mathrm{~K}]$ & $(1.01 \pm 0.05) \times 10^{7}$ & $(1.93 \pm 0.10) \times 10^{7}$
\end{tabular}

There are no experimental values of the elastic constants $c_{\mathrm{el}}$ for these ferroelectric materials in the literature as yet. Therefore, the obtained theoretical elastic constants $c_{\mathrm{el}}^{(1)}$ and $c_{\mathrm{el}}^{(2)}$, in two different ways, cannot be compared to any adequate experimental quantities.

\section{Conclusions}

The coupling of the spontaneous polarization $\langle\sigma\rangle$ (generally the primary order parameter) with the mechanical strain $\varepsilon$ (generally the secondary order parameter) causes that the primary order parameter $\langle\sigma\rangle$ gains the saturation region much faster than it would happen without the presence of the order parameters coupling [16, 17].

The dependence of the secondary order parameter on the primary order parameter follows from the equations of state in the compressible pseudospin model. Therefore, one can correctly identify right physical quantity as the secondary order parameter. Moreover, obtaining the agreement between experimental and theoretical curves of the secondary order parameter confirms that the compressible pseudospin model can be used to describe the behaviour of real materials.

The values of the elastic constant $c_{\mathrm{el}}$ can be obtained in two different ways as it has been done in Sect 4. As it has been shown, one needs only experimental data of the spontaneous polarization $\langle\sigma\rangle$ and of the spontaneous strain $\varepsilon$ to obtain the elastic constant $c_{\mathrm{el}}$ and a form of the coupling function $J(\varepsilon)$.

\section{Acknowledgments}

We thank Grażyna Bator and Przemysław Szklarz for valuable discussions. This work was supported by the Committee for Scientific Research (Poland): project No. N N204 147837.

\section{References}

[1] E.K.H. Salje, B. Wruch, H. Thomas, Z. Phys. B 82, 399 (1991).

[2] E.K.H. Salje, Phys. Rep. 215, 49 (1992).
[3] E.K.H. Salje, Phase Transition in Ferroelastic and Co-elastic Crystals, Cambridge University Press, Cambridge 1990.

[4] M.E. Lines, A.M. Glass, Principles and Applications of Ferroelectrics and Related Materials, Oxford, Clarendon Press 2004.

[5] J.J. Biney, N.J. Dowrick, A.J. Fisher, M.E.J. Newman, The Theory of Critical Phenomena, Clarendon, Oxford 1992.

[6] B. Widom, J. Chem. Phys. 43, 3898 (1965).

[7] R.B. Griffiths, Phys. Rev. 158, 176 (1967).

[8] M. Gałązka, Ph.D. Thesis, Institute of Nuclear Physics, Cracow 2006 (in Polish).

[9] M. Gałązka, P. Szklarz, G. Bator, P. Zieliński, J. Phys., Condens. Matter 18, 7145 (2006).

[10] P. Szklarz, M. Gałązka, G. Bator, P. Zieliński, Phys. Rev. B 74, 184111 (2006).

[11] F.J. Romero, M.C. Gallardo, J. Jiménez, J. del Cerro, J. Phys., Condens. Matter 17, 5001 (2005).

[12] P. Carpentier, Ph.D. Thesis, University of Science and Technology, Lille I 1995 (in French).

[13] M. Gałązka, P. Zieliński, Solid State Sci. 11, 740 (2009).

[14] M. Gałazka, P. Zieliński, P. Szklarz, G. Bator, Phase Transit. 80, 745 (2007).

[15] T. Sekido, T. Mitsui, J. Phys. Chem. Solids 28, 967 (1967).

[16] P. Carpentier, J. Lefebvre, R. Jakubas, W. Zając, P. Zieliński, Phase Transit. 67, 571 (1991).

[17] M. Gałązka, Phase Transit. 81, 1125 (2008).

[18] M. Gałazka, P. Szklarz, G. Bator, P. Zieliński, Solid State Phenom. 112, 141 (2006).

[19] M. Iwata, T. Tojo, T. Atake, Y. Ishibashi, J. Phys. Soc. Jpn. 63, 3751 (1994).

[20] J. Dziedzic, J. Mróz, R. Jakubas, Acta Cryst. Pol. A 108, 505 (2005).

[21] J. Lefebvre, P. Carpentier, R. Jakubas, Acta Crystallogr. B 47, 228 (1991).

[22] B.V. Andriyevsky, O. Ya. Maschchyshyn, M.O. Romanyuk, Condens. Matter Phys. 2, 693 (1999).

[23] R. Cach, R. Jakubas, Ferroelectrics 108, 121 (1990).

[24] M. Gałązka, P. Szklarz, G. Bator, P. Zieliński, Phase Transit. 79, 545 (2006).

[25] R. Jakubas, Solid State Commun. 69, 267 (1989).

[26] W. Rehwald, Adv. Phys. 22, 721 (1973).

[27] R. Jakubas, P. François, J. Lefebvre, Acta Phys. Pol. A 85, 579 (1994).

[28] B. Mróz, J.A. Tuszyński, H. Kiefte, M.J. Klouter, R. Jakubas, D. Sept, Phys. Rev. B 58, 14261 (1998).

[29] J. Przesławski, J. Phys., Condens. Matter 7, 4169 (1995).

[30] Cz. Pawlaczyk, K. Planta, Ch. Bruch, J. Stephan, H.-G. Unruh, J. Phys., Condens. Matter 4, 2687 (1992). 